\title{
EXTENSION OF UNIFORM MEASURES
}

\author{
ERROL CABY
}

\begin{abstract}
Necessary and sufficient conditions for a uniform measure to have a tight extension are presented. From these results, conditions for the factors of a tight measure to be also tight are derived.
\end{abstract}

1. Introduction. In this paper we derive conditions for a uniform measure $\mu$ on a uniform space $(\mathcal{X}, \mathscr{Q})$ to have an extension which is tight on $(\mathcal{X}, \mathcal{T})$, where $\mathcal{T}$ is a topology which is finer than the uniform topology of $\mathscr{U}$ and where $(\mathfrak{X}, \mathcal{T})$ is Hausdorff. These conditions are expressed in terms of the images of $\mu$ in certain complete metric spaces associated with $(\mathfrak{X}, \mathscr{Q})$. One consequence is that conditions for the factors of a tight measure to be also tight can be obtained.

In $\$ 2$ relevant properties of uniform measures are given, and a necessary and sufficient condition for extending a uniform measure as a tight measure is obtained. In $\$ 3$ we consider the following question. Let $E$ be a topological vector space. Let $\mu$ be a tight measure and $\nu_{1}, \nu_{2}$ be uniform measures on $E$ such that $\mu=\nu_{1} * \nu_{2}$. When are $\nu_{1}$ and $\nu_{2}$ tight? Finally, in $\S 4$, we consider the case where $(\mathcal{X}, \mathcal{T})$ is a complete metrizable linear space. Here we study the extent to which a condition, which is necessary and sufficient for a cylindrical measure on a Frechet space to have a tight extension, works for uniform measures. It should be noted that if $E$ is a locally convex space equipped with its weak topology, then the class of uniform measures is identical to the class of cylindrical measures.

2. General case. Let ( $(X, \mathcal{Q} L)$ be a uniform space, the class of uniform measures is defined as follows. Let $\Delta(\mathcal{X}, \mathcal{Q})$ denote the space of all bounded uniformly continuous real-valued functions on ( $\chi, \mathscr{Q}$ ) equipped with the supremum norm. Let $\Delta^{*}(\mathcal{X}, \mathscr{Q})$ be the dual of $\Delta(\mathcal{X}, \mathscr{Q})$, and $M_{s}$ the space of measures with finite support on $\mathcal{X}$. The class of uniform measures on ( $X, \mathcal{U})$, denoted by $M_{u}(\mathfrak{X}, \mathcal{Q})$, is defined as the closure of $M_{s}$ in $\Delta^{*}(\mathfrak{X}, \mathcal{Q})$ for the topology of uniform convergence on the uniformly equicontinuous and bounded subsets of $\Delta(\mathfrak{X}, \mathcal{Q})$.

Some of the basic properties of uniform measures are given next and can be found in $[2,3$ and 8$]$.

PROPOSITION 2.1. If $(\mathfrak{X}, \mathscr{Q})$ is a complete metric space, then $M_{u}(\mathfrak{X}, \mathscr{Q})$ is identical with $M_{t}(\mathcal{X})$, the class of bounded tight measures on $\mathcal{X}$.

Received by the editors February 25, 1983.

1980 Mathematics Subject Classification. Primary 28C15, 46G12, 60B05; Secondary 28C20.

Key words and phrases. Uniform space, uniform measures, cylindrical measures.

C1983 American Mathematical Society $0002-9939 / 83 \$ 1.00+\$ .25$ per page 
With the aid of the following definition, uniform measures on a general uniform space can be characterized in terms of their images in certain complete metric spaces.

Definition. Let $\mathscr{D}$ be a family of pseudometrics on a set $\mathcal{X}$. We shall say that $\mathscr{D}$ is directed if $\rho_{1}, \rho_{2} \in \mathscr{D}$ implies the existence of a $\rho_{3} \in \mathscr{D}$ such that for all $x$ and $y \in \mathcal{X}$

$$
\max \left\{\rho_{1}(x, y), \rho_{2}(x, y)\right\} \leqslant \rho_{3}(x, y)
$$

Let $\rho$ be a pseudometric on a set $\mathfrak{X}$, we shall let $\mathfrak{X}_{\rho}$ denote the metric quotient of the pseudometric space ( $\mathfrak{X}, \rho)$ and $\hat{X}_{\rho}$ denote the completion of $\mathfrak{X}_{\rho}$. In addition, we shall let $\pi_{\rho}$ denote the quotient map of $\mathcal{X}_{\text {into }} \hat{X}_{\rho}$.

Proposition 2.2. Let ( $\mathcal{X}, \mathcal{Q})$ be a uniform space and let $\mathscr{D}$ be a directed family of pseudometrics which generates $\mathscr{Q}$. Let $\mu \in \Delta^{*}(\mathfrak{X}, \mathcal{U})$. Then $\mu$ is a uniform measure if and only if $\pi_{\rho} \mu \in M_{t}\left(\hat{\chi}_{\rho}\right)$ for each $\rho \in \mathscr{D}$.

Using Proposition 2.2 we readily can deduce that $M_{\tau} \subset M_{u}(\mathfrak{X}, \mathscr{Q})$, where $M_{\tau}$ denotes the class of $\tau$-smooth functionals in $\Delta^{*}(\mathcal{X}, \mathcal{Q})$. It should be noted that there are spaces such that $M_{\tau}$ is strictly contained in $M_{u}$. Examples of such spaces have been given by LeCam and Maharam, see [8].

Definition. Let $\mathscr{D}$ be a family of pseudometrics on a set $\mathcal{X}$. A family of tight measures $\left\{\mu_{\rho}\right\}_{\rho \in \mathcal{D}}$ is said to be consistent if (1) $\mu_{\rho}$ is a measure on $\hat{\mathcal{X}}_{\rho}$; and (2) $\pi_{\rho^{\prime} \rho} \mu_{\rho}=\mu_{\rho^{\prime}}$ when $\rho^{\prime} \leqslant \rho$, where $\pi_{\rho^{\prime} \rho}$ is the quotient mar from $\hat{X}_{\rho}$ to $\hat{X}_{\rho^{\prime}}$.

Let $(\mathcal{X}, \mathcal{U})$ be a uniform space which is generated by a directed family of pseudometrics, $\mathscr{D}$, and let $\left\{\mu_{\rho}\right\}_{\rho \in \mathcal{D}}$ be a consistent family of tight measures. It is not difficult to see that there is a unique uniform measure $\mu$ on $(\mathcal{X}, \mathscr{U}$ ) such that $\pi_{\rho} \mu=\mu_{\rho}$ for each $\rho \in \mathcal{D}$.

REMARK. One consequence of the above is that it allows us to view metric space valued stochastic processes as uniform measures on convenient function spaces. For example, let $\left\{X_{t}, 0 \leqslant t \leqslant 1\right\}$ be a stochastic process with values in a complete, separable and metrizable linear space $E$. Then $\left\{X_{t}, 0 \leqslant t \leqslant 1\right\}$ can be regarded as a uniform measure on the space of continuous mappings of $[0,1]$ into $E, C([0,1] ; E)$, endowed with the uniformity of pointwise convergence. The question of whether this process has continuous paths can now be seen to be equivalent to the question of whether the associated uniform measure can be extended as a tight measure on $C([0,1] ; E)$ equipped with the topology of uniform convergence.

Having seen some of the basic properties of uniform measures, let us derive a necessary and sufficient condition for a uniform measure to have a tight extension. First we need the following result which can be found in [6, p. 135].

The setting is as follows. $I$ is a directed set, $\left(\mathscr{X}_{i}\right)_{i \in I}$ is a family of completely regular topological spaces and $\left(\mathfrak{f}_{i j}\right)_{i<j}$ is a family of continuous mappings: $\mathfrak{X}_{j} \rightarrow \mathfrak{X}_{i}$, satisfying $\mathfrak{f}_{i j} \mathfrak{f}_{j k}=\mathfrak{f}_{i k}$. We are given a family $\left(\mu_{i} \in M_{t}\left(\chi_{i}\right)\right)_{i \in I}$ of measures satisfying $f_{i j}\left(\mu_{j}\right)=\mu_{i}$, together with a topological space $\mathcal{O}$ and a family $\left(f_{i}\right)_{i \in I}$ of continuous mappings: $\mathscr{Y} \rightarrow \mathfrak{X}_{i}$ such that $\mathfrak{f}_{i j} \mathfrak{f}_{j}=\mathfrak{f}_{i}$ whenever $i<j$. 
THEOREM 2.3. There exists $\mu \in M_{t}(\mathcal{Y})$ such that $\mathfrak{f}_{i}(\mu)=\mu_{i}$ for each $i \in I$ if and only if $\sup _{i \in I}\left\|\mu_{i}\right\|<\infty$ and, given $\varepsilon>0$, there exists a compact $K_{\varepsilon}$ in $\mathcal{Y}$ such that $\left|\mu_{i}\right|\left(\mathfrak{X}_{i}-\mathfrak{f}_{i}\left(K_{\varepsilon}\right)\right)<\varepsilon$ for each $i$. If the mappings $\mathfrak{f}_{i}$ separate the points of $\mathcal{Y}, \mu$ is unique.

The desired result follows immediately and is stated below.

THEOREM 2.4. Let ( $\chi, \mathcal{Q})$ be a uniform space and let $\mathscr{Q}$ be a directed family of pseudometrics which generates $\mathcal{Q}$. Let $\mathcal{T}$ be a topology on $\mathcal{X}$ such that $(\mathfrak{X}, \mathcal{T})$ is Hausdorff and such that $\mathcal{T}$ is finer than the uniform topology of $\mathscr{Q}$. A uniform measure $\mu$ on $(\mathfrak{X}, \mathcal{Q})$ has a tight extension on $(\mathfrak{X}, \mathcal{T})$ if and only if for $\varepsilon>0$, there exists a compact $K_{\varepsilon}$ in $(\mathfrak{X}, \mathcal{T})$ such that $\left(\pi_{\rho} \mu\right)\left(\hat{X}_{\rho}-\pi_{\rho} K_{\varepsilon}\right)<\varepsilon$ for each $\rho \in \mathscr{D}$. If $(\mathfrak{X}, \mathcal{Q})$ is Hausdorff, the extension is unique.

3. Factors of a tight measure. Let $\mathcal{X}$ be a Hausdorff topological vector space with $\mathcal{Q}$ the associated translation-invariant uniformity. For $f \in \Delta(\mathfrak{X}, \mathcal{Q})$ and $x \in \mathcal{X}$, let $\tau_{x} f(y)=f(x+y)$. Now if $\nu \in \Delta^{*}(\mathcal{X}, \mathscr{Q})$, then $\nu\left(\tau_{x} f\right)$ is a bounded uniformly continuous function and, therefore, if $\mu$ is another functional in $\Delta^{*}(\mathcal{X}, \mathcal{Q})$ we can form the expression $\mu\left[\nu\left(\tau_{x} f\right)\right]$. This expression defines a continuous linear functional on $\Delta(\mathfrak{X}, \mathscr{Q})$ and can be used to define the convolution of $\mu$ and $\nu$ (for example, see [7, p. 262]). That is, $(\mu * \nu)(f)=\mu\left[\nu\left(\tau_{x} f\right)\right]$. However, when convolution is defined in this manner it need not be commutative on $\Delta^{*}$. This drawback, among other reasons, led LeCam in [9] to invent uniform measures, a class of linear functionals on which the above convolution operation is commutative.

In this section we shall derive conditions for the uniform factors of a tight measure to be tight. It should be noted that it is possible for a tight measure to have factors which are not tight. This can be seen from the following example.

EXAMPLE. Let $\mathcal{X}$ be the set of rational numbers with the usual metric $\rho$. Let $\left\{x_{n}\right\}$ and $\left\{y_{n}\right\}$ be sequences in $\mathcal{X}$ which converge to $\sqrt{2}$ and $1-\sqrt{2}$, respectively. Then linear functionals $\mu_{1}$ and $\mu_{2}$ can be defined on $\Delta(\mathcal{X}, \rho)$ as follows: $\mu_{1}(f)=$ $\lim _{n \rightarrow \infty} f\left(x_{n}\right)$ and $\mu_{2}(f)=\lim _{n \rightarrow \infty} f\left(y_{n}\right), f \in \Delta(X, \rho)$. It is clear that $\mu_{1}$ and $\mu_{2}$ belong to $M_{u}$ but not to $M_{t}$, and that $\mu_{1} * \mu_{2}=\delta_{1}$, where $\delta_{1}$ is the tight measure with unit mass at 1.

Definition. A linear functional $\mu$ on $\Delta(\mathfrak{X}, \mathcal{Q})$ will be called a mean if $\mu \geqslant 0$ (that is $\mu(f) \geqslant 0$ for $f \geqslant 0$ ) and $\mu(1)=1$. If a linear functional is both a mean and a uniform measure it will be called a uniform mean.

LEMMA 3.1. Let $\mathfrak{X}$ be a Hausdorff topological vector space (not necessarily locally convex). Let $\mu$ be a tight probability measure on $\mathcal{X}$ and $\nu_{1}, \nu_{2}$ uniform means on $\mathcal{X}$, such that $\mu=\nu_{1} * \nu_{2}$.

(a) If $\nu_{1}$ is tight then $\nu_{2}$ is also tight.

(b) If $\nu_{1}$ is a symmetrized mean then $\nu_{1}$ and $\nu_{2}$ are both tight.

Proof. (a) Let $\varepsilon>0$. Since $\mu$ and $\nu_{1}$ are tight we can find compact subsets of $\mathcal{X}$, $K$ and $K^{\prime}$, such that $\mu(K) \geqslant 1-\varepsilon / 2$ and $\nu_{1}\left(K^{\prime}\right) \geqslant 1-\varepsilon / 2$. 
Let $\mathscr{Q}$ be a directed family of pseudometrics compatible with the vector space operations which generates the topology on $\mathcal{X}$. Let $\rho \in \mathscr{D}$, then $\pi_{\rho} \mu, \pi_{\rho} \nu_{1}$ and $\pi_{\rho} \nu_{2}$ are tight measures on $\hat{\mathfrak{X}}_{\rho}$ and $\pi_{\rho} \mu=\left(\pi_{\rho} \nu_{1}\right) *\left(\pi_{\rho} \nu_{2}\right)$. Therefore,

$$
\begin{aligned}
1-\varepsilon / 2 & \leqslant \pi_{\rho} \mu\left(\pi_{\rho} K\right)=\int_{\hat{\mathfrak{X}}_{\rho}} \pi_{\rho} \nu_{2}\left(\pi_{\rho} K-x\right) \pi_{\rho} \nu_{1}(d x) \\
& =\int_{\pi_{\rho} K^{\prime}} \pi_{\rho} \nu_{2}\left(\pi_{\rho} K-x\right) \pi_{\rho} \nu_{1}(d x)+\int_{\hat{\mathfrak{x}}_{\rho}-\pi_{\rho} K^{\prime}} \pi_{\rho} \nu_{2}\left(\pi_{\rho} K-x\right) \pi_{\rho} \nu_{1}(d x) \\
& \leqslant \int_{\pi_{\rho} K^{\prime}} \pi_{\rho} \nu_{2}\left(\pi_{\rho} K-\pi_{\rho} K^{\prime}\right) \pi_{\rho} \nu_{1}(d x)+\int_{\hat{\hat{X}_{\rho}}-\pi_{\rho} K^{\prime}} 1 \pi_{\rho} \nu_{1}(d x) \\
& \leqslant \pi_{\rho} \nu_{2}\left(\pi_{\rho}\left(K-K^{\prime}\right)\right)+\varepsilon / 2 .
\end{aligned}
$$

Thus $\pi_{\rho} \nu_{2}\left(\pi_{\rho}\left(K-K^{\prime}\right)\right) \geqslant 1-\varepsilon$ and so, by Theorem $2.4, \nu_{2}$ is tight on $\chi$.

(b) Suppose $\nu_{1}$ is a symmetrized uniform mean, that is $\nu_{1}=\lambda * \bar{\lambda}$, where $\lambda, \bar{\lambda} \in M_{u}$ and $\bar{\lambda}(f)=\lambda(\bar{f}), \bar{f}(x)=f(-x)$. Since $\mu$ is tight we can find a compact $K$ such that $\mu(K) \geqslant 1-\varepsilon / 2$.

Let $\rho$ be in $\mathscr{D}$. Then

$$
1-\varepsilon / 2 \leqslant \pi_{\rho} \mu\left(\pi_{\rho} K\right)=\int_{\hat{\hat{\chi}_{\rho}}} \pi_{\rho} \lambda\left(\pi_{\rho} K-x\right) \pi_{\rho}\left(\bar{\lambda} * \nu_{2}\right)(d x),
$$

from which it follows that there exists $x^{\prime} \in \hat{\mathcal{X}}_{\rho}$ such that $\pi_{\rho} \lambda\left(\pi_{\rho} K-x^{\prime}\right) \geqslant 1-\varepsilon / 2$.

Hence, $\pi_{\rho}(\lambda * \bar{\lambda})\left(\pi_{\rho}(K-K)\right) \geqslant 1-\varepsilon$ and, therefore, $\nu_{1}=\lambda * \bar{\lambda}$ is tight by Theorem 2.4. From (a) it follows that $\nu_{2}$ is also tight.

REMARK. This lemma and the following Corollary generalize some results for cylindrical measures which were obtained by Tortrat in [10]. Note that if $E$ is a locally convex space endowed with its weak topology then the class of uniform measures is the same as the class of cylindrical measures.

COROllary. Let $\mathcal{X}$ and $\mathscr{Q}$ be as above and let $\mu_{1}$ and $\mu_{2}$ be tight measures on $\mathcal{X}$. If for each $\rho \in \mathscr{D}$ we can find an $x_{\rho} \in \hat{\mathscr{X}}_{\rho}$ such that $\pi_{\rho} \mu_{1}=\delta_{x_{\rho}} * \pi_{\rho} \mu_{2}$ then there exists an $x \in \mathcal{X}$ such that $x_{\rho}=\pi_{\rho} x$ for each $\rho$ and $\mu_{1}=\delta_{x} * \mu_{2}$.

To prove this result we need the following fact which can be found in [4].

LEMMA 3.2. Let $\mu$ be a tight probability measure on a Hausdorff topological vector space $\mathfrak{X}$. If $\delta_{x} * \mu=\delta_{y} * \mu$, where $x$ and $y$ are in $\mathcal{X}$, then $x=y$.

Proof of the Corollary. Let $\rho, \rho^{\prime}$ be in $\mathscr{Q}$ with $\rho^{\prime} \leqslant \rho$. Then

$$
\pi_{\rho^{\prime} \rho}\left(\delta_{x_{\rho}} * \pi_{\rho} \mu_{2}\right)=\pi_{\rho^{\prime} \rho}\left(\pi_{\rho} \mu_{1}\right)=\pi_{\rho^{\prime}} \mu_{1}=\delta_{x_{\rho^{\prime}}} *\left(\pi_{\rho^{\prime}} \mu_{2}\right) \text {. }
$$

Therefore,

$$
\delta_{\pi_{\rho^{\prime} \rho} x_{\rho}} *\left(\pi_{\rho^{\prime}} \mu_{2}\right)=\delta_{x_{\rho^{\prime}}} *\left(\pi_{\rho^{\prime}} \mu_{2}\right)
$$

By Lemma 3.2 we get $\pi_{\rho^{\prime} \rho} x_{\rho}=x_{\rho^{\prime}}$ and, therefore, $\left\{\delta_{x_{\rho}}\right\}_{\rho \in \mathcal{D}}$ is consistent. Hence, we can find a $\nu \in M_{u}$ such that $\pi_{\rho} \nu=\delta_{x_{\rho}}$. Lemma 3.1 now implies that $\nu$ is tight. 
To complete the proof we need to show that the support of $\nu$ consists of a single point. Suppose we can find two distinct points $y$ and $z$ in the support of $\nu$. Then we can find a $\rho \in \mathscr{D}$ such that $\rho(y-z)>0$. Furthermore, since $y$ and $z$ belong to the support of $\nu$,

$$
\nu\left(\left\{u ; u \in \mathcal{X} \text { and } \rho(u-y)<\varepsilon_{1}\right\}\right)>0
$$

and

$$
\nu\left(\left\{u ; u \in \mathcal{X} \text { and } \rho(u-z)<\varepsilon_{2}\right\}\right)>0
$$

for any $\varepsilon_{1}>0$ and $\varepsilon_{2}>0$. If we let $y_{\rho}$ and $z_{\rho}$ denote the points $\pi_{\rho} y$ and $\pi_{\rho} z$ in $\hat{X}_{\rho}$, respectively, it is clear that $y_{\rho} \neq z_{\rho}$ and $\pi_{\rho} \nu\left(\left\{w ; w \in \hat{X}_{\rho}\right.\right.$ and $\left.\left.\rho\left(w-y_{\rho}\right)<\varepsilon_{1}\right\}\right)>0$ and $\pi_{\rho} \nu\left(\left\{w ; w \in \hat{X}_{\rho}\right.\right.$ and $\left.\left.\rho\left(w-z_{\rho}\right)<\varepsilon_{2}\right\}\right)>0$ for any $\varepsilon_{1}>0$ and $\varepsilon_{2}>0$. This leads to a contradiction since $\pi_{\rho} \nu$ has unit mass concentrated at the single point $x_{\rho}$. Hence the Corollary.

4. Complete metrizable linear spaces. Let $F$ be a Fréchet space and let $m$ be a cylindrical measure on $F$. It is known (see [1, p. 100]) that $m$ extends to a tight measure on $F$ if and only if for each $\varepsilon>0$, there exists a finite $A \subset F$ such that $m^{*}(\{y:|y-A| \leqslant \varepsilon\}) \geqslant 1-\varepsilon$, where $m^{*}(B)=\inf \{m(A) ; A$ is a cylindrical set on $F$ and $A \supset B\}$. We shall now try to discover the extent to which this type of condition works for extending uniform measures. First, however, we need another definition and some preliminary results.

DEFINITION. Let $\mathcal{X}$ be a topological space and let $\mathscr{D}$ be a family of continuous pseudometrics on $\mathcal{X}$. If $\left\{A_{i}\right\}_{i \in I}$ is a nonempty family of subsets of $\mathcal{X}$ such that $A=\cap_{i \in I} A_{i} \neq \varnothing$, then $\left\{A_{i}\right\}_{i \in I}$ is said to be adapted with respect to $\mathscr{D}$ if for each $\rho \in \mathscr{D}$ one has $\overline{\pi_{\rho} A} \supset \bigcap_{i \in I} \overline{\pi_{\rho} A_{i}}$ and thus, $\overline{\pi_{\rho} A}=\bigcap_{i \in I} \overline{\pi_{\rho} A_{i}}$, where $\overline{\pi_{\rho} A}$ and $\overline{\pi_{\rho} A_{i}}$ are the closures of $\pi_{\rho} A$ and $\pi_{\rho} A_{i}$ in $\hat{X}_{\rho}$, respectively.

LEMMA 4.1. Let $\mathfrak{X}$ be a topological vector space. Let $\mathfrak{T}$ be a neighborhood base of zero and $K$ a compact subset of $\mathcal{X}$. Then the family $(K+V)_{V \in \mathfrak{X}}$ is adapted with respect to $\mathscr{D}$, where $\mathcal{D}$ is any family of uniformly continuous pseudometrics compatible with the vector space operations.

Proof. Let $\rho \in(1)$. Since $\pi_{\rho}: \mathcal{X} \rightarrow \hat{X}_{\rho}$ is continuous, each open ball in $\hat{X}_{\rho}$ with center 0 contains a $\pi_{\rho} V, V \in \mathfrak{X}$. Thus each closed ball in $\hat{\mathcal{X}}_{\rho}$ with center 0 contains a $\overline{\pi_{\rho} V}, V \in \mathcal{K}$. Let $S_{\rho}(\varepsilon)$ denote the closed ball in $\hat{\mathcal{X}}_{\rho}$ with radius $\varepsilon$ and center 0 . Then

$$
\begin{aligned}
\bigcap_{V \in \mathcal{R}} \overline{\pi_{\rho}(K+V)} & =\bigcap_{V \in \mathcal{R}}\left(\pi_{\rho} K+\overline{\pi_{\rho} V}\right) \subset \bigcap_{\varepsilon>0}\left(\pi_{\rho} K+S_{\rho}(\varepsilon)\right) \\
& =\pi_{\rho} K=\overline{\pi_{\rho} K}
\end{aligned}
$$

Now $K=\bigcap_{V \in \Re}(K+V)$. Therefore, $\bigcap_{V \in \Re} \overline{\pi_{\rho}(K+V)} \subset \overline{\pi_{\rho}\left[\bigcap_{V \in \Re}(K+V)\right]}$ and, thus, $\{K+V\}_{V \in \mathscr{N}}$ is adapted. 
Lemma 4.2. Let $\mathfrak{X}$ be a complete Hausdorff topological vector space and let $\mathfrak{X}$ be a neighborhood base of symmetric closed neighborhoods of zero. For each $V \in \mathfrak{\Re}$, let $K_{V}$ be a compact subset of $\mathcal{X}$ and suppose that $K=\bigcap_{V \in \mathfrak{X}}\left(K_{V}+V\right) \neq \varnothing$. Then

(a) $K$ is compact, and

(b) for every neighborhood $U$ of zero, there exists a finite subset $\mathcal{F}$ of $\mathscr{N}$ such that $K+U$ contains $\bigcap_{V \in \mathscr{F}}\left(K_{V}+V\right)$.

Proof. See [1, p. 97].

LEMMA 4.3. Let ( $\mathscr{X}, \mathcal{Q})$ be a uniform space and let $\mathscr{D}$ be a family of uniformly continuous pseudometrics on $\mathcal{X}$. Let $\left\{A_{i}\right\}$ be a decreasing family of subsets of $\mathcal{X}$ which is adapted with respect to $\mathscr{D}$. Let $\mu$ be a uniform mean on $(\mathcal{X}, \mathcal{Q})$. Then if $\left(\pi_{\rho} \mu\right) \overline{\left(\pi_{\rho} A_{i}\right)} \geqslant 1-\varepsilon$ for each $\rho \in \mathscr{D}$ and each $i \in I$, one has $\left(\pi_{\rho} \mu\right) \overline{\left(\pi_{\rho} A\right)} \geqslant 1-\varepsilon$ for each $\rho \in \mathscr{D}$, where $A=\bigcap_{i \in I} A_{i}$.

Proof. The proof is similar to that of Theorem III, 5; 1 on p. 79 of [1]. It is based on the fact that $\pi_{\rho} \mu$ is a tight measure on $\hat{\mathcal{X}}_{\rho}$ for each $\rho \in \mathscr{D}$ and that $\left\{\overline{\pi_{\rho} A_{i}}\right\}_{i \in I}$ is a decreasing family of closed subsets whose intersection is $\overline{\pi_{\rho} A}$.

The following result can now be derived from the above results and Theorem 2.4. The proof is quite similar to that of Theorem IV, 2; 1 on p. 99 of [1].

THEOREM 4.4. Let $(\mathfrak{X},||)$ be a complete metrizable linear space. Let $\mathcal{T}^{\prime}$ be a vector topology on $\mathcal{X}$ which is coarser than the topology of $\|$. Suppose that $\sigma^{\prime}$ is generated by $\mathscr{D}$, a directed family of pseudometrics compatible with the vector space operations. Then a uniform measure $\mu$ on $\left(\mathfrak{X}, \mathcal{T}^{\prime}\right)$ has an extension as a tight measure on $(\mathfrak{X}, \|)$ if and only if for each $\varepsilon>0$ there exist finite subsets $A_{k}^{\varepsilon} \subset \mathcal{X}, k \geqslant 1$, such that

$$
\bigcap_{k=1}^{\infty}\left(A_{k}^{\varepsilon}+S(1 / k)\right) \neq \varnothing
$$

and such that, for each $\rho \in \mathscr{D},\left(\pi_{\rho} \mu\right) *\left(\pi_{\rho}\left[\cap_{k=1}^{n}\left(A_{k}^{\varepsilon}+S(1 / k)\right)\right]\right) \geqslant 1-\varepsilon$ for every $n \geqslant 1$, where $S(\delta)=\{y ;|y| \leqslant \delta, y \in \mathcal{X}\}$. The extension is unique if $\left(\mathcal{X}, \mathcal{J}^{\prime}\right)$ is Hausdorff.

It would appear that in order to replace the condition in the above theorem by one equivalent to that given for extending cylindrical measures on Fréchet spaces at the beginning of this section, it would be necessary to make additional assumptions about $\mathcal{T}^{\prime}$ and $\|$.

\section{REFERENCES}

1. A. Badrikian and S. Chevet, Mesures cylindriques, espaces de Wiener et fonctions aleatoires Gaussiennes, Springer-Verlag, Berlin and New York, 1974.

2. E. Caby, Convergence of measures on uniform spaces, Thesis, Univ. of California, 1976.

3. __ The weak convergence of uniform measures, J. Multivariate Anal. 9(1979), 130-137.

4. I. Csiszar and B. Rajput, $\boldsymbol{A}$ convergence of types theorem for probability measures on topological vector spaces with applications to stable laws, Z. Wahrsch. Verw. Gebiete 36 (1976), 1-7.

5. R. Dudley, J. Feldman and L. LeCam, On seminorms and probabilities, and abstract Wiener spaces, Ann. of Math. (2) 93 (1971), 390-408.

6. D. Fremlin, D. Garling and R. Haydon, Bounded measures on topological spaces, Proc. London Math. Soc. (3) 25 (1972), 115-136. 
7. E. Hewitt and K. Ross, Abstract harmonic analysis, Vol 1, Springer-Verlag, Berlin and New York, 1979.

8. L. LeCam, Note on a certain class of measures, unpublished.

9. __ Remarques sur le théorème limite central dans les espaces localement convexes, Les Probabilitès sur les Structures Algébriques, Editions CNRS, Paris, 1970, pp. 233-249.

10. A. Tortrat, Structure des lois indéfiniment divisibles dans un espace vectoriel topologique (séparé) $X$ (Sympos. Probability Methods in Analysis), Lecture Notes in Math., vol. 31, Springer-Verlag, Berlin, 1967, pp. 299-328.

Department of Mathematics, Howard University, Washington, D.C. 20059

Current address: Department of Statistics and Probability, Michigan State University, East Lansing, Michigan 48824 